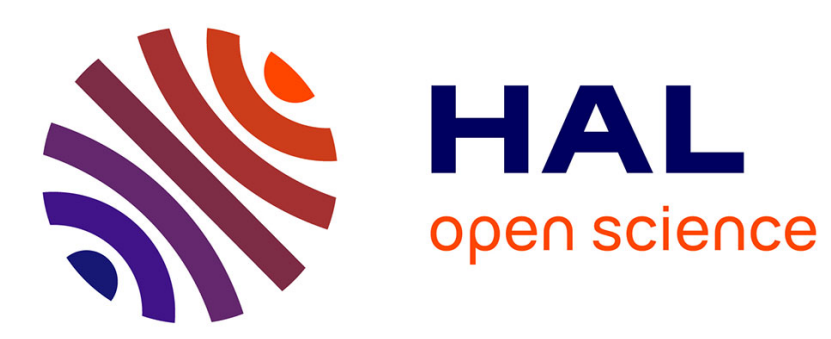

\title{
The inferior fronto-occipital fascicle: a century of controversies from anatomy theaters to operative neurosurgery.
}

Alessandro de Benedictis, Carlo Efisio Marras, Laurent Petit, Silvio Sarubbo

\section{- To cite this version:}

Alessandro de Benedictis, Carlo Efisio Marras, Laurent Petit, Silvio Sarubbo. The inferior frontooccipital fascicle: a century of controversies from anatomy theaters to operative neurosurgery.. Journal of Neurosurgical Sciences, 2021. hal-03345943

\section{HAL Id: hal-03345943 \\ https://hal.science/hal-03345943}

Submitted on 16 Sep 2021

HAL is a multi-disciplinary open access archive for the deposit and dissemination of scientific research documents, whether they are published or not. The documents may come from teaching and research institutions in France or abroad, or from public or private research centers.
L'archive ouverte pluridisciplinaire HAL, est destinée au dépôt et à la diffusion de documents scientifiques de niveau recherche, publiés ou non, émanant des établissements d'enseignement et de recherche français ou étrangers, des laboratoires publics ou privés. 
The inferior fronto-occipital fascicle: a century of controversies from anatomy theaters to operative neurosurgery

Running title: The inferior fronto-occipital fascicle

ALESSANDRO DE BENEDICTIS ${ }^{1 *}$, CARLO EFISIO MARRAS ${ }^{1}$, LAURENT PETIT ${ }^{2}$ and SILVIO SARUBBO ${ }^{3}$

${ }^{1}$ Neurosurgery Unit, Department of Neurosciences, Bambino Gesù Children's Hospital, IRCCS, Rome, Italy;

${ }^{2}$ Groupe d'Imagerie Neurofonctionnelle, Institut Des Maladies Neurodégénératives, UMR 5293, CNRS, CEA University of Bordeaux, Bordeaux, France;

3 Division of Neurosurgery, Structural and Functional Connectivity Lab, S. Chiara Hospital, Trento, Italy.

*Corresponding author: Alessandro De Benedictis, MD, PhD Neurosurgery Unit, Department of Neurosciences, Bambino Gesù Children's Hospital, IRCCS, 4, piazza S. Onofrio, 00165, Rome, Italy. E-mail: alessandro.debenedictis@opbg.net 


\begin{abstract}
INTRODUCTION: Since its first description in the early $19^{\text {th }}$ century, the inferior frontooccipital fascicle (IFOF) and its anatomo-functional features were neglected in the neuroscientific literature for the last century. In the last decade, the rapid development of in vivo imaging for the reconstruction of white matter (WM) connectivity (i.e., tractography) and the consequent interest in more traditional ex vivo methods (postmortem dissection) have allowed a renewed debate about course, termination territories, anatomical relationships, and functional roles of this fascicle.
\end{abstract}

EVIDENCE ACQUISITION: We reviewed the main current knowledge concerning the structural and functional anatomy of the IFOF and possible implications in neurosurgical practice.

EVIDENCE SYNTHESIS: The IFOF connects the occipital cortex, the temporo-basal areas, the superior parietal lobule, and the pre-cuneus to the frontal lobe, passing through the ventral third of subinsular WM of the external capsule. This wide distribution of cortical terminations provides multimodal integration between several functional networks, including language, non-verbal semantic processing, object identification, visuo-spatial processing and planning, reading, facial expression recognition, memory and conceptualization, emotional and neuropsychological behavior. This anatomo-functional organization has important implication also in neurosurgical practice, especially when approaching the frontal, insular, temporo-parieto-occipital regions and the ventricular system.

CONCLUSIONS: The IFOF is the most extensive associative bundle of the human connectome. Its multi-layer organization reflects important implications in many aspects of brain functional processing. Accurate awareness of IFOF functional anatomy and integration between multimodal datasets coming from different sources has crucial implications for both neuroscientific knowledge and quality of neurosurgical treatments.

Key words: inferior fronto-occipital fascicle; white matter; tractography; neurosurgery; brain mapping. 


\section{THE HISTORY}

The first substantial contribution to the anatomical characterization of fronto-occipital connectivity is attributed to the German neuroanatomist K.F. Burdach in 1822. Early observations made by J. Reil in 1809 described the existence of a WM tract covering the ventral temporo-occipital region, the so-called inferior longitudinal fascicle (ILF). Burdach clearly distinguished the ILF from the uncinate fascicle (UF) and identified a component of the ILF passing through the "floor" of the EC, to reach the lateral cortex of the frontal lobe. ${ }^{1}$

Ignored by Déjerine in his description of long association fibers, ${ }^{2}$ a fronto-occipital fascicle passing along the claustrum was differentiated from the ILF by P. Trolard in 1906: "It seems to us, in short, that in the region we have just considered, there are two distinct fascicles, although they both end in the occipital lobe. In support of this way of seeing, we shall not only invoke their separation so easily obtained with the help of our preparation, but we shall rely above all on their anterior termination, which is quite different. The first belongs to the frontal lobe, while the second belongs to the temporal lobe". ${ }^{3}$

In 1909, Edward Curran highlighted the final architecture of the IFOF, including termination territories within the frontal, occipital, parietal, and temporal lobes and relationships with basal ganglia, namely the lentiform nucleus (LN). Moreover, he introduced the crucial concept of "stem", which consists of the segment in which fibers' direction and compactness are clearly evident and distinguishable from the surrounding anatomical structures. ${ }^{4}$

Despite these seminal notions, subsequent research was quite discontinuous over the $19^{\text {th }}$ century and until the first decade of the third millennium. ${ }^{5-15}$

A renewed interest for the anatomical investigation on the inferior fronto-occipital fascicle (IFOF) derived from the development of tractography and brain mapping techniques in patients who underwent resection of infiltrative tumors located in eloquent areas, especially in language regions. The systematic and reproducible elicitation of semantic paraphasias (i.e. the substitution of a word with another that is conceptually related to the intended word) by electrostimulation in awake conditions further characterized the anatomical substrate related to intraoperative functional responses. ${ }^{16-23}$ Following this evidence, Martino et al. in 2010 applied the traditional Klingler technique 
to reproduce the anatomical IFOF course and showed the multilayer organization of this pathway, especially within the posterior territories. ${ }^{13}$

Indeed, the debate on the IFOF was influenced by anatomical studies in animals. Previous authors questioned the existence of IFOF in the rhesus monkey because it was not identified with the gold standard tracing methods. Therefore, they proposed that the IFOF observed in tractography in humans may be due to a diffusion-weighted artifact explained by the proximity of the ILF with the UF and the extreme capsule (ExC). ${ }^{24,25}$

However, this was not confirmed by at least three subsequent studies. In fact, by directly comparing tractography results, Mars et al. in 2016 showed a convincing anatomic similarity between the macaque and human connections, suggesting the presence of a common ventral pathway directly connecting the frontal and occipital lobes. ${ }^{26}$ Moreover, Decramer et al. in 2018 demonstrated the existence of IFOF in rhesus monkey brains through post-mortem dissection. ${ }^{27}$ Finally, in a recent study combining post-mortem diffusion MRI tractography with Klingler micro-dissection in the same brain, our group provided further evidence for the existence of bilateral fiber tracts in non-human primates (Old-World vervet monkeys) that corresponded to the human IFOF in terms of trajectory, topological organization, and cortical terminal fields. Demonstration of a ventral pathway in the primate lineage, potentially supporting ability for semantic elaboration, comprehension, and syntax communication, was important to understanding the anatomical background for evolution of language and social behavior. ${ }^{28}$

As a result, continuous methodological advancements and integration of anatomical data provided by ex vivo techniques and non-invasive functional imaging methods (in particular tractography) are contributing to progressively refining knowledge of the IFOF, especially concerning topography of terminations, organization of different subcomponents, relationships with other white matter (WM) pathways, and functional implications of the different components (Figure 1)..$^{29-39}$

\section{STRUCTURAL AND FUNCTIONAL ANATOMY}

\section{Structural anatomy}

The anatomical characterization of the IFOF has been debated by previous authors, concerning not only the existence, but also the exact topography of its terminations within the anterior and the posterior brain territories (Figure 2). Although cortical fibers have been historically attributed to the inferior frontal and fronto-orbital cortices and the ventral 
occipital cortex, recent results from post-mortem dissection and in vivo tractography studies showed that fiber distribution is wider than previously reported (Figure 2A). ${ }^{13,38-40}$

Better results were obtained through a new approach based on adopting the pathway stem (i.e., the point of passage where all fibers pass through) as starting anatomical reference for both dissection and tractography analysis. This method allows easier tract identification, with both dissection and tractography techniques, and more accurate annotation, especially concerning terminations territories, intra-subject and interindividual variabilities, and asymmetries. ${ }^{41}$

Following this approach, after removing the insular cortex, the IFOF stem is found at the level of the limen insulae within the antero-ventral third of the external capsule (EC). Although strictly related, fibers belonging to the IFOF stem (dorsal-posterior) are separated from the UF stem (ventral-anterior) (Figure 2B,C). From this point, the IFOF fibers follow a fan-shaped development in both the anterior and posterior directions.

Within the frontal lobe, two layers, superficial and deep, are identified. Superficial fibers arch antero-superiorly and medio-laterally at the level of the superior limiting sulcus of the insula and reach the cortices of the pars triangularis $(\mathrm{pTr})$ and the pars opercularis (pOp) of the inferior frontal gyrus (IFG) (Figure 2D).

Deep terminations pass under the inferior frontal sulcus (IFS) to reach, from posterior to anterior, the dorso-lateral prefrontal cortex (DLPFC), including the middle third of the middle frontal gyrus (MFG), the lateral and anterior OFCs, and the fronto-basal cortices (Figure 2D,E). ${ }^{38}$

Within the posterior regions, the IFOF follows an inferior-lateral and posterior course from the limen insulae, along the roof of the temporal horn and the lateral surface of the atrium and the occipital horn of the lateral ventricle, in the context of the sagittal stratum (SS). Along their course, fibers progressively enlarge to describe a $45^{\circ}$ angle, which leads to a wide distribution range within the parietal, occipital and temporal cortices (Figure $2 \mathrm{~F}) .^{22}$

As for the anterior portion, a superficial and a deep layer are also identifiable for the posterior segment. Superficial fibers are connected to the convexity surface of the superior parietal lobule (SPL) and the posterior portion of the superior occipital gyrus (SOG) and the middle occipital gyrus (MOG). The deep components terminate into the posterior part of the inferior occipital gyrus (IOF) and the following regions in the occipital and the posterior and basal region of the temporal lobe, including: the posterior portion of the 
fusiform gyrus (FuG), the temporo-occipital sulcus, and the basal surface of the inferior temporal gyrus (ITG) (Figure 2F,G). ${ }^{13,35}$

Following diffusion weight imaging (DWI) studies confirmed the multilayer distribution of IFOF fibers. ${ }^{42,43}$ A recent study based on tractography analysis in 20 healthy adult subjects confirmed the anterior distribution of IFOF cortical terminations over the inferior frontal cortex, the prefrontal cortex, the orbito-frontal cortex (OFC), and the frontal pole, and within the occipital (cuneus, lateral occipital gyri, and lingual gyrus), SPL, and temporal cortices in posterior territories (ITG, middle temporal gyrus, and FuG) (Figure 3). Interestingly, a significant inter-hemispheric asymmetry was also found, with IFOF projections' prevalence to the SPL in the right hemisphere. In contrast, terminations within the IFG resulted in leftward lateralization. ${ }^{40}$

\section{Functional anatomy}

The complex multilayer organization of the IFOF and the widespread distribution of its cortical terminations form the basis of this fascicle's involvement in several aspects of functional processing.

The superficial component connects the SPL, the occipital extra-striate cortex, the Wernicke's territories, and the fusiform area at the occipito-temporal junction to the $\mathrm{p} \operatorname{Tr}$ and the pOp of the IFG. These regions are crucially involved in the language network. It has been demonstrated that the IFOF mediates the semantic elaboration of language by integrating visual information coming from posterior territories with meaning processing occurring within the frontal region. ${ }^{18}$ The role of IFOF in the direct sematic ventral stream of language has been confirmed by direct subcortical stimulation (DES) in patients who underwent resection of brain infiltrating tumors during awake surgery with awake monitoring. DES systematically induces the systematic elicitation of semantic paraphasia or anomia during intraoperative object naming task. ${ }^{16-19}$

The statistical analysis of these subcortical functional responses in the most extensive series never reported demonstrated a distribution of semantic errors concordant with the anatomical course of the IFOF from the deep WM of the temporal lobe to the frontal lobe, over the roof of the temporal horn of the ventricle and in the most ventral portion of the EC, below the anterior third of the insula. ${ }^{34,36,37}$ Frontal terminations to the DLPFC and the posterior MFG agree with the role of these regions in the semantic elaboration of language, as demonstrated by imaging and electrostimulation studies. ${ }^{16,44,45}$ 
Moreover, DES studies demonstrated that IFOF fibers stimulation during non-verbal semantic association tests is associated with reproducible impairments within both dominant and non-dominant hemispheres. ${ }^{46,47}$ These findings have been confirmed by the probabilistic analysis of distributions of a more extensive set of responses to DES, showing the existence of a bilateral network subserving the non-verbal semantic system and a crucial role of the IFOF of the "non-dominant" hemisphere for language elaboration in the non-verbal semantic processing. ${ }^{36}$

The extensive fronto-parieto-occipital connectivity of the IFOF underlies the integration of complex movements, perception, and location of body parts between sensory-motor, occipital areas, and output frontal centers (namely the ventro-lateral prefrontal cortex) involved in object identification, visual concentration, and planning of visually guided movements. ${ }^{29}$ For example, lesion studies in patients suffering from neglect due to cortical damages or disconnection mechanisms indicate that the right IFOF, especially the deep parieto-frontal component, participates in visuo-spatial processing. ${ }^{48,49}$

Involvement of IFOF in reading has been suggested by analyzing age-related differences in the tensor metrics in dyslexic patients compared with normal reading subjects. ${ }^{50,51}$

The IFOF is also involved in emotional facial expression recognition, in the ability to remember new faces and conceptualization, through connections to the FuG and the OFC, as revealed in patients with congenital and acquired prosopagnosia. ${ }^{52-57}$ Finally, the correlation between IFOF degeneration in patients with antisocial personality disorder and obsessive-compulsive disorder indicated the role of IFOF in neuropsychological behavior. ${ }^{58}$

\section{IFOF AND NEUROSURGERY}

Interest in investigating the IFOF functional anatomy was not limited to fundamental research purposes, but increasingly also involved the neurosurgical community. In fact, as described in the previous section, essential aspects concerning the role of the IFOF in many neurocognitive domains, such as language, have been highlighted by using direct cortical and subcortical mapping during surgical procedures for brain gliomas. ${ }^{16,20}$

Moreover, instead of the cortical epicenters, the limited plastic potential of WM implies that subcortical connections should be carefully considered and preserved during resection, and especially at the level of the region of a high density of fibers, such as the 
stem. In fact, preservation of the main WM bundles reduces the risk of permanent postsurgical deficits, due to both local direct damage of the fascicle and postdisconnection consequences to distant regions. ${ }^{16}$

These considerations make IFOF a notably critical structure for at least two reasons: first, its particularly long course over all the lobes of the human brain and, second, the high-concentration of fibers in a relatively small stem located within a high-risk area for brain surgery, that is the anterior third of the subinsular WM.

Consequently, a growing body of literature indicates that the systematic application of a network-based approach in neurosurgical practice, overcoming the classical localizationist model of a strict region-function association, has a positive impact on the safety and efficacy of surgical procedures, even in regions traditionally deemed to have higher rates of permanent deficits. For this reason, several authors emphasized the role of an accurate and systematic preoperative anatomical characterization of the IFOF course and intraoperative electrical mapping to evaluate relationships with the target, and to plan the best tailored surgical strategy according to eloquent subcortical boundaries. ${ }^{16,59-62}$

In the lateral frontal region, for lobectomy or extensive lesionectomy within the dominant hemisphere, IFOF is identified by eliciting semantic paraphasias and/or anomia at the level of the postero-lateral subcortical territory underneath the IFG. ${ }^{21}$ Within this area, IFOF fibers show a notable degree of crossing and interdigitation with terminations of the superior longitudinal fascicle (SLF III), namely at the level of the IFG-IFS, the MFG, and the DLPFC. As reported by neuroimaging and stimulation studies, this fiber overlapping reflects the wide distribution of activations belonging to specific aspects of language elaboration (phonemic, semantic, and syntactic). ${ }^{21,35,63,64}$

Accurate awareness of the IFOF stem and its course within the temporal stem is crucial for planning insular resections through both trans-opercular or trans-sylvian routes. In the case of trans-opercular subpial approach, after resection of the pTr of the IFG or, more generally, the frontal portion of the tumor, fibers of IFOF stem running within the frontal WM constitute the medial functional limit the surgical WM corridor to access the insula (Figure 4). ${ }^{65-67}$

The IFOF constitutes, in fact, the medial and deep functional limit of resection within the temporal stem in the case of trans-sylvian resection. The IFOF stem in this region is not exclusively an important functional reference, but also an anatomo-vascular landmark, being, in fact, the last layer of WM before the territory of the lenticulo-striate arteries. ${ }^{66}$ 
Thus, DES exploration for identifying the IFOF stem in this region can be useful even in the non-dominant hemisphere for language processing.

The temporal portion of the IFOF has been indicated as an additional and reliable landmark in case of temporal resection during oncologic or epilepsy surgery, in both dominant hemisphere for language semantic processing (commonly tested with object naming), and for non-verbal semantics (commonly tested with palm-pyramid-tree test) within the non-dominant hemisphere. ${ }^{46,68}$ In particular, the IFOF constitutes the posterosuperior and deep limit of resection in the posterior portion of the temporal pole, along the roof of the temporal horn, and, more anteriorly, the supero-mesial and deep boundary at the roof of the tip of the temporal horn, where IFOF fibers converge to enter the stem with the EC. ${ }^{69}$

It is worth noting that identifying by DES the whole course of the IFOF in the WM of the temporal lobe implies preserving the superior portion of the optic radiation (OR). Thus, preservation of semantic stream during temporal resection is crucial also to avoid postoperative impairments to visual field..$^{70,71}$

Anatomical dissection analysis of the three-dimensional relationships of the IFOF at the level of the temporal stem revealed that the fascicle crosses the posterior two-thirds of the temporal stem, within the space between the posterior limit of the UF $(8 \mathrm{~mm}$ from the limen insulae) and the lateral geniculate body, passing above the OR and below the auditory radiations, claustro-opercular fibers, and insulo-opercular fibers of the EC and the ExC. These subcortical functional landmarks can be adopted to integrate classic cortical surgical limits, improving the safety of surgical approaches to this region, such as the posterior trans-sylvian approach to mesio-temporal structures and the temporal transopercular approach to the insula. ${ }^{65}$ In a recent work, the impact of temporal epilepsy surgery on long-range connectivity, including the IFOF, has been confirmed by simulating different possible approaches to the temporo-mesial area, ranging from standard resections to more focused minimally-invasive techniques. ${ }^{72}$

The temporo-parieto-occipital junction is another region where IFOF has to be carefully considered when planning resection. In fact, this region is involved in several eloquent neurological aspects, including language, visuo-spatial, writing, and other highlevel functions. ${ }^{22,39,73,74}$ This area is crossed by an intricate network of WM pathways responsible for integrating semantic and sensory information. Seven long-distance tracts, forming the so-called temporo-parietal fiber intersection area, have been described, 
including the SLF III and SLF II, the arcuate fascicle (AF), the middle longitudinal fasciculus (MdLF), the ILF, the IFOF, the OR, and the tapetum. ${ }^{39}$ Concerning specifically the IFOF, recent works have characterized its anatomical course within this region and have highlighted its complex relationships with other fascicles. ${ }^{22,39}$ In particular, the IFOF has a horizontal course anterior-to-posterior in the SS context, partially overlapping the superior component of the OR until the tracts diverge to reach their respective anterior and posterior termination areas. ${ }^{22,35,70}$ The ILF runs inferiorly and laterally to the OR and the IFOF, with an anterior and inferior direction to temporo-basal cortices. At the temporooccipital junction, IFOF fibers cross medially the AF, the posterior SLF, and, more posterior, the vertical occipital fascicle. ${ }^{22,75}$ For this reason, the surgical approach to ventral lesions that involves the temporal and occipital territories should be tailored according to the horizontal orientation of associative pathways running within this area, namely the IFOF, the ILF, and the OR.

Finally, the wide distribution of IFOF fibers implies a strict relationship with the ventricular system. In fact, at the level of the most anterior and ventral portion of the EC, the IFOF covers along its course the roof of the temporal horn, the lateral wall of the atrium, and the occipital horn. At the temporal stem, the tract overlaps the entire superior OR and the superior third of the inferior OR. ${ }^{65,70}$ The surgical approach to the ventricles is challenging, mainly due to their deep location, the possible morphological variability, the relationships with the dense layer of parallel WM tracts which cover the whole ventricular wall from anterior to posterior, and the proximity to critical neural structures, such as the thalamus and the internal capsule. For these reasons, many authors have discussed the best surgical corridors to reach the target without damaging the eloquent subcortical connectivity. ${ }^{76-78}$

The IFOF is involved in the anterior frontal transcortical, anterior temporal, and posterior trans-temporal approaches. ${ }^{76}$ The anterior frontal transcortical approach allowed access to the frontal horn, the anterior part of the body of the lateral ventricle, and the anterior and superior aspects of the third ventricle. Beyond the IFOF, the main WM tracts running in this region are the anterior part of the SLF II, the AF, the corona radiata fibers and ventral callosal fibers, and SLF I and the cingulum more medially. Consequently, the main possible risks associated with surgical damage of subcortical connections include language disorders, limbic system dysfunction, and impairments of spatial awareness. ${ }^{76,77}$ 
When approaching the temporal horn, in addition to the IFOF, the OR, the AF, the anterior commissure, the UF, and the ILF should be considered, with potential postoperative development of visual deficits, naming problems, and memory deficits. ${ }^{76}$

Finally, in the posterior trans-temporal approaches to the middle or posterior third of the temporal horn and the atrium, the main WM structures associated with the IFOF are the SLF II, the AF, the MdLF, the anterior commissure, and the OR. In these cases, potential postsurgical neurological sequelae include anomia, phonemic semantic troubles, visual deficits, and Gerstman syndrome. ${ }^{76}$

\section{CONCLUSIONS AND FUTURE PERSPECTIVES}

Although its debated and controversial neuro-anatomical history, the IFOF is currently acknowledged as one of the most important components of the brain connectome.

Methodological advances have allowed for the progressive characterization the IFOF as a complex and multilayer bundle, connecting several distant distributed cerebral territories (occipito-extrastriate, temporo-basal, superior parietal, and frontal) involved in many language and non-language functions.

Moreover, the combined use of traditional dissections with modern neuroimaging and mapping techniques have further contributed to validating previous evidence and highlighting new anatomo-functional aspects. In this context, DES of WM tracts represents, so far, the best way to provide accurate information on the effective cerebral connectomic anatomy of the IFOF and to explore the dynamic interactions between different neural subcircuits and possible regulation mechanisms by other subnetworks.

In parallel with scientific interest, knowledge of IFOF functional anatomy is also mandatory for neurosurgical practice, to plan the best approach to specific targets, improve intraoperative orientation, and minimize the risk of postsurgical permanent and disabling deficits. In this context, as showed by previous experience on the SLF/AF complex, brain photogrammetry represents a novel tool allowing a layer-by-layer threedimensional representation of WM anatomy coming from a real specimen. This method opens the door to many possible applications, including interactive visualization of WM tracts, quantitative information acquisition, and post-dissection analysis combined with MRI data. Implementing brain photogrammetry to the IFOF and other bundles will further improve this technique's value for neuroscientific, education, and neurosurgical purposes. $^{32}$ 
In the same vein, development of a functional probabilistic atlas is of particular interest as a complementary tool to existing neuroimaging techniques (fMRI, TMS, DTI, MEG), to obtain a more detailed perspective of the WM functional anatomy, concerning critical structures of individual patients and their evolution over the time. ${ }^{34,36,79,80}$

Finally, systematic merging of a multimodal dataset (including probabilistic atlases, digitalized anatomical data, structural and functional neuroimaging data at the individual and population level) is crucial to improve the anatomical characterization of critical pathways of the central nervous system and to understand functional processes served by WM bundles at both long-range and short-range, not only to improve neuroscientific knowledge, but also to optimize preoperative planning and surgical results.

\section{REFERENCES}

1. Burdach KF. Vom Baue und Leben des Gehirns. Leipzig: Dyk; 1822.

2. Dejerine, J., \& Dejerine-Klumpke, A. (1895). Anatomie des Centres Nerveux. Paris: Rueff et Cie; 1895. p.749-783.

3. Trolard P. Le faisceau longitudinal inférieur du cerveau. Revue Neurologique $1906 ; 14: 440-446$.

4. Curran EJ. A new association fiber tract in the cerebrum with remarks on the fiber tract dissection method of studying the brain. J Comp Neurol Psychol 1909;19:645656.

5. Davis LE. An anatomic study of the inferior longitudinal fasciculus. Archives of Neurology and Psychiatry 1921;5:370e381.

6. Hultkrantz W. Gehirnpraeperation mittels Zerfaserung. Anleitung zum makroskopischen Studium des Gehirns. Berlin: Verlag von Julius Springer; 1929.

7. Elliott CH. Textbook of the Nervous System. A Foundation for Clinical Neurology. Philadelphia: J.B. Lippincott Company; 1947.

8. Crosby E, Humphrey T, and Lauer E. Correlative Anatomy of the Nervous System. New York: Macmillan; 1962.

9. Ebeling $U$ and von Cramon D. Topography of the uncinate fascicle and adjacent temporal fiber tracts. Acta Neurochirurgica 1992;115:43e148.

10. Kier EL, Staib LH, Davis LM, Bronen RA. MR imaging of the temporal stem: Anatomic dissection tractography of the uncinate fasciculus, inferior occipitofrontal 
fasciculus, and Meyer's loop of the optic radiation. American Journal of Neuroradiology 2004;25:677e691.

11. Fernandez-Miranda JC, Rhoton Jr AL, Alvarez-Linera J, Kakizawa Y, Choi C, de Oliveira EP. Three-dimensional microsurgical and tractographic anatomy of the white matter of the human brain. Neurosurgery 2008;62:989e1026.

12. Lawes IN, Barrick TR, Murugam V, Spierings N, Evans DR, Song M, et al. Atlasbased segmentation of white matter tracts of the human brain using diffusion tensor tractography and comparison with classical dissection. NeuroImage 2008;39:62e79.

13. Martino J, Brogna C, Robles SG, Vergani F, Duffau H. Anatomic dissection of the inferior fronto-occipital fasciculus revisited in the lights of brain stimulation data. Cortex 2010;46:691-699.

14. Martino J, De Witt Hamer P, Vergani F, Brogna C, de Lucas EM, Vazquez-Barquero A, et al. Cortex-sparing fiber dissection: An improved method for the study of white matter anatomy in the human brain. Journal of Anatomy 2011;219:531e541.

15. Forkel SJ, Thiebaut de Schotten M, Kawadler JM, Dell'Acqua F, Danek A, Catani M. The anatomy of fronto-occipital connections from early blunt dissections to contemporary tractography. Cortex 2014;56:73-84.

16. Duffau H, Gatignol P, Mandonnet E, Peruzzi P, Tzourio-Mazoyer N, Capelle L. New insights into the anatomo-functional connectivity of the semantic system: A study using cortico-subcortical electrostimulations. Brain 2005;128:797-810

17. Duffau H, Peggy Gatignol ST, Mandonnet E, Capelle L, Taillandier L. Intraoperative subcortical stimulation mapping of language pathways in a consecutive series of 115 patients with Grade II glioma in the left dominant hemisphere. Journal of Neurosurgery 2008;109:461-471

18. Duffau H, Moritz-Gasser S, Mandonnet E. A re-examination of neural basis of language processing: proposal of a dynamic hodotopical model from data provided by brain stimulation mapping during picture naming. Brain Lang 2014;131:1-10.

19. Duffau H. Stimulation mapping of white matter tracts to study brain functional connectivity. Nat Rev Neurol 2015;11:255-265.

20. De Benedictis A, Duffau H. Brain hodotopy: from esoteric concept to practical surgical applications. Neurosurgery 2011;68:1709-1723.

21. De Benedictis A, Sarubbo S, Duffau H. Subcortical surgical anatomy of the lateral frontal region: human white matter dissection and correlations with functional insights 
provided by intraoperative direct brain stimulation: laboratory investigation. $\mathrm{J}$ Neurosurg 2012;117:1053-1069.

22. De Benedictis A, Duffau H, Paradiso B, Grandi E, Balbi S, Granieri E, et al. Anatomo-functional study of the temporo-parieto-occipital region: dissection, tractographic and brain mapping evidence from a neurosurgical perspective. $\mathrm{J}$ Anat 2014;225:132-151.

23. Sierpowska J, Gabarrós A, Fernández-Coello A, Camins À, Castañer S, Juncadella M, et al. White-matter pathways and semantic processing: intrasurgical and lesionsymptom mapping evidence. Neuroimage Clin 2019;22:101704.

24. Schmahmann JD, Pandya DN. Fiber pathways of the brain. New York: Oxford University Press; 2006.

25. Schmahmann JD, Pandya DN, Wang R, Dai G, D’Arceuil HE, de Crespigny AJ, et al. Association fibres pathways of the brain: parallel observations from diffusion spectrum imaging and autoradiography. Brain 2007;130:630-653.

26. Mars RB, Foxley S, Verhagen L, Jbabdi S, Sallet J, Noonan MP, et al. The extreme capsule fiber complex in humans and macaque monkeys: a comparative diffusion MRI tractography study. Brain Struct Funct 2016;221:4059-4071.

27. Decramer T, Swinnen S, van Loon J, Janssen P, Theys T. White matter tract anatomy in the rhesus monkey: a fiber dissection study. Brain Struct Funct 2018;223:36813688 .

28. Sarubbo S, Petit L, De Benedictis A, Chioffi F, Ptito M, Dyrby TB. Uncovering the inferior fronto-occipital fascicle and its topological organization in non-human primates: the missing connection for language evolution. Brain Struct Funct 2019;224:1553-1567.

29. Aralamask A, Ulmer JL, Kocak M, Salvan CV, Hillis AE, Youssen DM. Association, commissural, and projection pathways and their functional deficit reported in literature. J Comput Assist Tomogr 2006;30:695-715.

30. Caverzasi E, Papinutto N, Amirbekian B, Berger MS, Henry RG. Q-ball of inferior fronto-occipital fasciculus and beyond. PLoS ONE 2014;9:e100274.

31. Zemmoura I, Serres B, Andersson F, Barantin L, Tauber C, Filipiak I, et al. FIBRASCAN: a novel method for $3 \mathrm{D}$ white matter tract reconstruction in MR space from cadaveric dissection. Neuroimage 2014;103:106-118. 
32. De Benedictis A, Nocerino E, Menna F, Remondino F, Barbareschi M, Rozzanigo U, et al. Photogrammetry of the human brain: a novel method for three-dimensional quantitative exploration of the structural connectivity in neurosurgery and neurosciences. World Neurosurg 2018;115:e279-e291.

33. Wu Y, Sun D, Wang Y, Wang Y. Subcomponents and connectivity of the inferior fronto-occipital fasciculus revealed by diffusion spectrum imaging fiber tracking. Front Neuroanat 2016;10:88.

34. Sarubbo S, De Benedictis A, Merler S, Mandonnet E, Balbi S, Granieri E, et al. Towards a functional atlas of human white matter. Hum Brain Mapp 2015;36:31173136.

35. Sarubbo S, De Benedictis A, Merler S, Mandonnet E, Barbareschi M, Dallabona M, et al. Structural and functional integration between dorsal and ventral language streams as revealed by blunt dissection and direct electrical stimulation. Hum Brain Mapp 2016;37:3858-3872.

36. Sarubbo S, Tate M, De Benedictis A, Merler S, Moritz-Gasser S, Herbet G, et al. Mapping critical cortical hubs and white matter pathways by direct electrical stimulation: an original functional atlas of the human brain. Neuroimage 2020;205:116237.

37. Sarubbo S, Tate M, De Benedictis A, Merler S, Moritz-Gasser S, Herbet G, et al. A normalized dataset of 1821 cortical and subcortical functional responses collected during direct electrical stimulation in patients undergoing awake brain surgery. Data Brief 2019;28:104892.

38. Sarubbo S, De Benedictis A, Maldonado IL, Basso G, Duffau H. Frontal terminations for the inferior fronto-occipital fascicle: anatomical dissection, DTI study and functional considerations on a multi-component bundle. Brain Struct Funct 2013;218:21-37.

39. Martino J, da Silva-Freitas R, Caballero H, de Lucas EM, García-Porrero JA, Vázquez-Barquero A. Fiber dissection and diffusion tensor imaging tractography study of the temporoparietal fiber intersection area. Neurosurgery $2013 ; 72: 87-97$.

40. Vassal F, Pommier B, Sontheimer A, Lemaire JJ. Inter-individual variations and hemispheric asymmetries in structural connectivity patterns of the inferior frontooccipital fascicle: a diffusion tensor imaging tractography study. Surg Radiol Anat 2018;40:129-137. 
41. Hau J, Sarubbo S, Perchey G, Crivello F, Zago L, Mellet E, et al. Cortical terminations of the inferior fronto-occipital and uncinate fasciculi: stem-based anatomical virtual dissection. Front Neuroanat 2016;10:58.

42. Conner AK, Briggs RG, Sali G, Rahimi M, Baker CM, Burks JD, et al. A connectomic atlas of the human cerebrum-Chapter 13: Tractographic Description of the Inferior Fronto-Occipital Fasciculus. Oper Neurosurg (Hagerstown) 2018;15:S436-S443.

43. Panesar SS, Yeh F-C, Deibert CP, Fernandes-Cabral D, Rowthu V, Celtikci P, et al. A diffusion spectrum imaging-based tractographic study into the anatomical subdivision and cortical connectivity of the ventral external capsule: uncinate and inferior frontooccipital fascicles. Neuroradiology 2017;59:971-987.

44. Sakai KL. Language acquisition and brain development. Science 2005;310:815-819.

45. Plaza M, Gatignol P, Cohen H, Berger B, Duffau H. A discrete area within the left dorsolateral prefrontal cortex involved in visual-verbal incongruence judgment. Cereb Cortex 2008;18:1253-1259.

46. Moritz-Gasser S, Herbet G, Duffau H. Mapping the connectivity underlying multimodal (verbal and non-verbal) semantic processing: a brain electrostimulation study. Neuropsychologia 2013;51:1814-1822.

47. Herbet G, Moritz-Gasser S, Duffau H. Direct evidence for the contributive role of the right inferior fronto-occipital fasciculus in non-verbal semantic cognition. Brain Struct Funct 2017;222:1597-1610.

48. Urbanski M, Thiebaut de Schotten M, Rodrigo S, Catani M, Oppenheim C, Touzé E, et al. Brain networks of spatial awareness: evidence from diffusion tensor imaging tractography. J Neurol Neurosurg Psychiatry. 2008;79:598-601.

49. Toba MN, Migliaccio R, Batrancourt B, Bourlon C, Duret C, Pradat-Diehl P, et al. Common brain networks for distinct deficits in visual neglect. A combined structural and tractography MRI approach. Neuropsychologia 2018;115:167-178.

50. Rollins NK, Vachha B, Srinivasan P, Chia J, Pickering J, Hughes CW, et al. Simple developmental dyslexia in children: alterations in diffusion-tensor metrics of white matter tracts at 3 T. Radiology 2009;251:882-891.

51. Motomura K, Fujii M, Maesawa S, Kuramitsu S, Natsume A, Wakabayashi T. Association of dorsal inferior frontooccipital fasciculus fibers in the deep parietal lobe with both reading and writing processes: a brain mapping study. J Neurosurg 2014;121:142-148. 
52. Dutton GN. Cognitive vision, its disorders and differential diagnosis in adults and children: knowing where and what things are. Eye (Lond) 2003;17:289-304.

53. Philippi CL, Mehta S, Grabowski T, Adolphs R, Rudruaf D. Damage to association fiber tracts impairs recognition of the facial expression of emotion. J Neurosci 2009;29:15089-15099.

54. Thomas C, Avidan G, Humphreys K, Jung K, Gao F, Behrmann M. Reduced structural connectivity in ventral visual cortex in congenital prosopagnosia. Nature Neuroscience 2009;12:29-31.

55. Valdés-Sosa M, Bobes MA, Quiñones I, Garcia L, Valdes-Hernandez PA, Iturria Y, et al. Covert face recognition without the fusiform-temporal pathways. Neuroimage 2011;57:1162-1176.

56. Comes-Fayos J, Romero-Martinez A, Moya-Albiol L. Role of major long fiber tracts association in empathy. Rev Neurol 2018;67:263-272.

57. Unger A, Alm KH, Collins JA, O'Leary JM, Olson IR. Variation in White Matter Connectivity Predicts the Ability to Remember Faces and Discriminate Their Emotions. J Int Neuropsychol Soc 2016;22:180-190.

58. Waller R, Dotterer HL, Murray L, Maxwell AM, Hyde LW. White-matter tract abnormalities and antisocial behavior: A systematic review of diffusion tensor imaging studies across development. NeuroImage 2017;14:201-215.

59. Duffau H. A two-level model of interindividual anatomo-functional variability of the brain and its implications for neurosurgery. Cortex 2017;86:303-313.

60. Henderson F, Abdullah KG, Verma R, Brem S. Tractography and the connectome in neurosurgical treatment of gliomas: the premise, the progress, and the potential. Neurosurg Focus 2020;48:E6.

61. Sarubbo S, Latini F, Sette E, Milani P, Granieri E, Fainardi E, et al. Is the resection of gliomas in Wernicke's area reliable?: Wernicke's area resection. Acta Neurochir (Wien) 2012;154:1653-1662.

62. Herbet G, Duffau H. Revisiting the Functional Anatomy of the Human Brain: Toward a Meta-Networking Theory of Cerebral Functions. Physiol Rev 2020;100:1181-1228.

63. Makris N, Kennedy DN, McInerney S, Sorensen AG, Wang R, Caviness VS Jr, et al. Segmentation of subcomponents within the superior longitudinal fascicle in humans: a quantitative, in vivo, DT-MRI study. Cereb Cortex 2005;15:854-869. 
64. Vigneau M, Beaucousin V, Hervé PY, Duffau H, Crivello F, Houdé O, et al. Metaanalyzing left hemisphere language areas: phonology, semantics, and sentence processing. Neuroimage 2006;30:1414-1432.

65. Martino J, Vergani F, Robles SG, Duffau H. New insights into the anatomic dissection of the temporal stem with special emphasis on the inferior fronto-occipital fasciculus: implications in surgical approach to left mesiotemporal and temporoinsular structures. Neurosurgery 2010;66:4-12.

66. Mandonnet E, Martino J, Sarubbo S, Corrivetti F, Bouazza S, Bresson D, et al. Neuronavigated fiber dissection with pial preservation: laboratory model to simulate opercular approaches to insular tumors. World Neurosurg 2017;98:239-242.

67. Pastor-Escartín F, García-Catalán G, Holanda VM, Muftah Lahirish IA, Quintero RB, Neto MR, et al. Microsurgical anatomy of the insular region and operculoinsular association fibers and its neurosurgical application. World Neurosurg 2019;129:407420.

68. Vilasboas T, Herbet G, Duffau H. Challenging the Myth of Right Nondominant Hemisphere: Lessons from Corticosubcortical Stimulation Mapping in Awake Surgery and Surgical Implications. World Neurosurg 2017;103:449-456.

69. Duffau H, Thiebaut de Schotten M, Mandonnet E. White matter functional connectivity as an additional landmark for dominant temporal lobectomy. J Neurol Neurosurg Psychiatry 2008;79:492-495.

70. Sarubbo S, De Benedictis A, Milani P, Paradiso B, Barbareschi M, Rozzanigo U, et al. The course and the anatomo-functional relationships of the optic radiation: a combined study with 'post mortem' dissections and 'in vivo' direct electrical mapping. J Anat 2015;226:47-59.

71. Kier EL, Staib LH, Davis LM, Bronen RA. MR imaging of the temporal stem: Anatomic dissection tractography of the uncinate fasciculus, inferior occipitofrontal fasciculus, and Meyer's loop of the optic radiation. American Journal of Neuroradiology 2004;25:677e691.

72. Busby N, Halai AD, Parker GJM, Coope DJ, Lambon Ralph MA. Mapping whole brain connectivity changes: The potential impact of different surgical resection approaches for temporal lobe epilepsy. Cortex 2019;113:1-14. 
73. Bullock D, Takemura H, Caiafa CF, Kitchell L, McPherson B, Caron B, et al. Associative white matter connecting the dorsal and ventral posterior human cortex. Brain Struct Funct 2019;224:2631-2660.

74. Palejwala AH, O' Connor KP, Pelargos P, Briggs RG, Milton CK, Conner AK, et al. Anatomy and white matter connections of the lateral occipital cortex. Surg Radiol Anat 2020;42:315-328.

75. Jitsuishi T, Hirono S, Yamamoto T, Kitajo K, Iwadate Y, Yamaguchi White matter dissection and structural connectivity of the human vertical occipital fasciculus to link vision-associated brain cortex. A. Sci Rep 2020;10:820.

76. Güngör A, Baydin S, Middlebrooks EH, Tanriover N, Isler C, Rhoton AL Jr. The white matter tracts of the cerebrum in ventricular surgery and hydrocephalus. $\mathrm{J}$ Neurosurg 2017;126:945-971.

77. Capilla-Guasch P, Quilis-Quesada V, Regin-Neto M, Holanda VM, González-Darder JM, de Oliveira E. White Matter Relationships Examined by Transillumination Technique Using a Lateral Transcortical Parietal Approach to the Atrium: ThreeDimensional Images and Surgical Considerations. World Neurosurg 2019;132:e783e794.

78. Muftah Lahirish IA, Middlebrooks EH, Holanda VM, Batista-Quintero R, Maeda FL, Neto MR, et al. Comparison between transcortical and interhemispheric approaches to the atrium of lateral ventricle using combined white matter fiber dissections and magnetic resonance tractography. World Neurosurg 2020;138:e478-e485.

79. Zacà D, Corsini F, Rozzanigo U, Dallabona M, Avesani $\mathrm{P}$, Annicchiarico L, et al. Whole-Brain Network Connectivity Underlying the Human Speech Articulation as Emerged Integrating Direct Electric Stimulation, Resting State fMRI and Tractography. Front Hum Neurosci 2018;12:405.

80. Zacà D, Jovicich J, Corsini F, Rozzanigo U, Chioffi F, Sarubbo S. ReStNeuMap: a tool for automatic extraction of resting-state functional MRI networks in neurosurgical practice. J Neurosurg 2018;131:764-771.

81. Mazoyer B, Mellet E, Perchey G, Zago L, Crivello F, Jobard G, et al. BIL\&GIN: A neuroimaging, cognitive, behavioral, and genetic database for the study of human brain lateralization. Neuroimage 2016;124:1225-1231. 
Conflicts of interest. The authors certify that there is no conflict of interest with any financial organization regarding the material discussed in the manuscript.

Funding. The authors report no involvement in the research by the sponsor that could have influenced the outcome of this work.

Authors' contributions. - All authors have given substantial contributions to the conception, the draft, and the final approval of the manuscript.

Acknowledgements. - The authors acknowledge the Sanitary Department of the Autonomous Province of Trento (Italy) and the Direction Team of the Azienda Sanitaria per i Servizi Sanitari of Trento for their support to research activities of the NeuSurPlan Proejct, and are grateful to Aalap Herur-Raman for the language editing of this manuscript.

\section{FIGURE LEGENDS}

Figure 1. Overview of the main methods developed to investigate the IFOF functional anatomy. (A) Early investigation by post-mortem WM dissection (adapted from Curran 1909); $;^{4}$ (B) Modern tractography allowed for improvement of the IFOF anatomical characterization in vivo and non-invasively; (C) Example of modern probabilistic functional atlas: sagittal and axial sequences showing the probability distribution of functional results collected by direct intraoperative electrical awake mapping during resection of brain tumors. Distribution of semantic responses, plotted and mapped onto volumetric MNI T1 sequences, correspond to the course of the IFOF (adapted from Sarubbo et al. 2020). ${ }^{36}$

Figure 2. Dissection of the IFOF within a left hemisphere, according to Klingler technique. (A) General overview of the IFOF (green), connecting the temporo-parietooccipital areas to the frontal lobe; (B) The IFOF stem (blue tag) is exposed within the anterior third of the infra-insular space, at the level of the limen insulae, after removal of the grey matter of the claustrum and the EC. From this point, the fibers enlarge with a vertical course in the anterior direction and more horizontal toward the posterior regions. The temporal ventricular horn has been exposed to show fibers running above the roof 
(red pin); (C) Further dissection of basal ganglia with exposition of the lentiform nucleus (LN). The IFOF stem (a) runs postero-dorsally to the UF (violet arrow); (D) Anterior termination of the IFOF from the stem $(a)$ to the frontal lobe. In addition to superficial fibers, reaching the pTr and pOp of the IFG (red arrow and circle), deeper fibers are identified running under the IFS (blue tags); (E) Overview of frontal terminations, according to their superficial course (red arrow and circle), and deeper distribution, from posterior to anterior, to the DLPFC (green arrow and circle), the MFG (light green arrow and circle), the orbito-frontal and the polar region (blue arrow and circles); (F) The posterior course of the IFOF is characterized by a progressive enlargement of fibers from the stem (blue $\operatorname{tag} a$ ) to the parietal (green arrow) and occipital cortices (violet arrow). Relationships with fibers of the corona radiata and the AF are also shown; (G) More ventral orientation of the specimen, showing the ventral temporo-basal fibers (pink arrow) (Original pictures from the dataset of the Department of Neurosurgery, Structural and Functional Connectivity Lab Project, Azienda Sanitaria per i Servizi Sanitari of Trento, Italy).

Figure 3. Tractography of a left IFOF. (A) Lateral; (B) Medial; (C) Superior; (D) Inferior view. Colors differentiate the different components, according to anatomical analysis described: superficial fibers connecting the temporo-parieto-occipital area to the IGF (red), deep fibers reaching the DLPFC and the MFG (green), deep portion direct to the to the lateral and basal orbitofrontal cortices (blue) (Original pictures from the tractography of the IFOF extracted from 411 whole-brain tractograms of the BIL\&GIN database). ${ }^{81}$

Figure 4. Surgical case, showing resection of a low-grade glioma located within the left dorsal insula. (A) Preoperative MRI showing, from up to down, coronal, sagittal and axial sequences. (B) Intraoperative picture showing the frontal transopercular access to the insular region (yellow circle). Direct electrical mapping in awake condition was adopted to identify the eloquent cortical and subcortical regions. In particular, a denomination test was used for language mapping (left superior box). At the cortical level, the eloquent sites were identified: ventral premotor cortex, evoking speech arrest when stimulated (tag 3), motor cortex of the face (tag 2), somato-sensory cortex (tag 1), MFG, evoking anomia when stimulated ( $\operatorname{tag} 2$ ). (C) Dissection of the frontal and insular region by Klingler technique. The specimen has been oriented according to the surgical perspective. After 
removal of the insular cortex, the IFOF stem is identified at the level of the limen insulae (green tag a) and fibers coming from the posterior region to the frontal lobe are exposed (pink arrows). The yellow circle corresponds to the infero-medial limit of subcortical resection (yellow arrow). At this level, IFOF fibers are identified by eliciting anomia at DES. Within the frontal lobe, deep IFOF fibers pass under the IFS (blue tags) and are crossed by the SLF III, running in the context of the IFG (IFG). (D) Postoperative MRI (from up to down, coronal and axial sequences), with probabilistic constrained spherical deconvolution tractography (60 Dir, $2.4 \mathrm{~mm}, 1.5 \mathrm{~T}$ scanner), showing the tumor resection and the main surrounding WM pathways, corresponding to the functional boundaries of resection, as detected at intraoperative subcortical DES. The superior functional limit was identified stimulating the fibers of the SLF III (green tract, green arrow), by evoking verbal apraxia. In the posterior part, the superior, lateral and medial limit was identified evoking tongue and face movements at stimulation of the pyramidal tract, arching to the ventral third of the motor area (blue tract). The infero-medial limit corresponded to the IFOF, by evoking anomia at DES. These fibers pass through the EC and enter the WM of the frontal lobe (red arrows, red tract) (Original pictures from the dataset of the Department of Neurosurgery, Structural and Functional Connectivity Lab Project, Azienda Sanitaria per i Servizi Sanitari of Trento, Italy). 


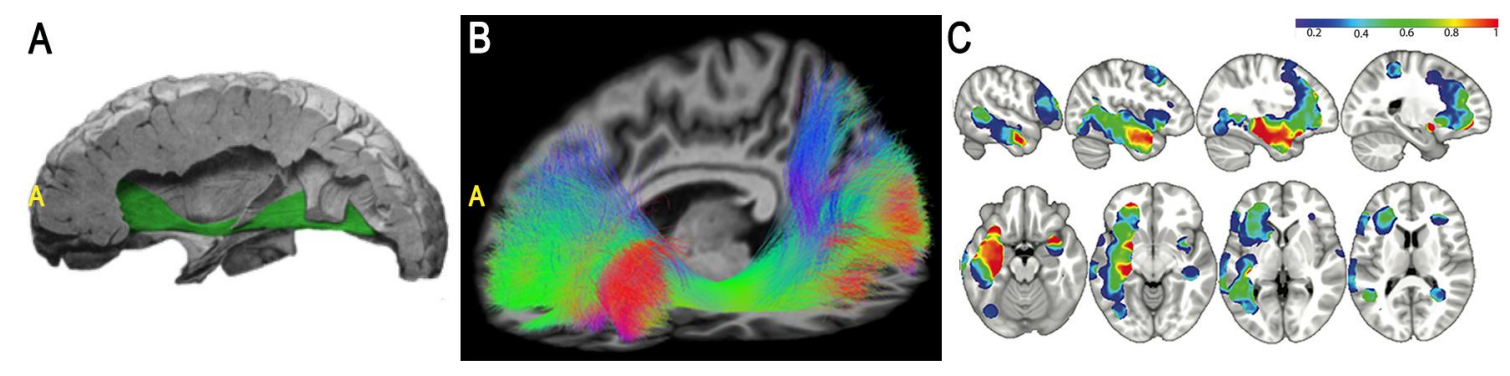



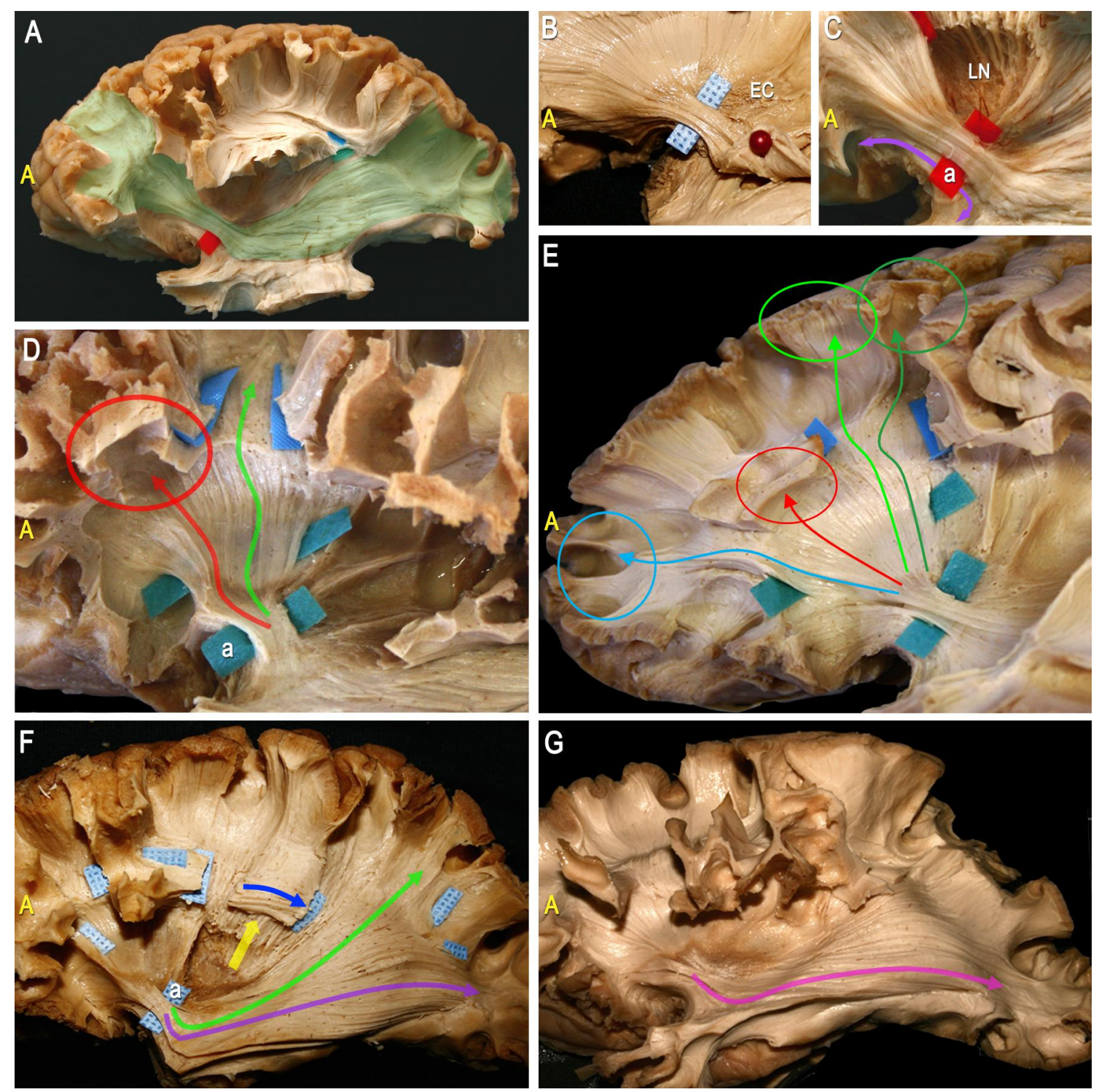


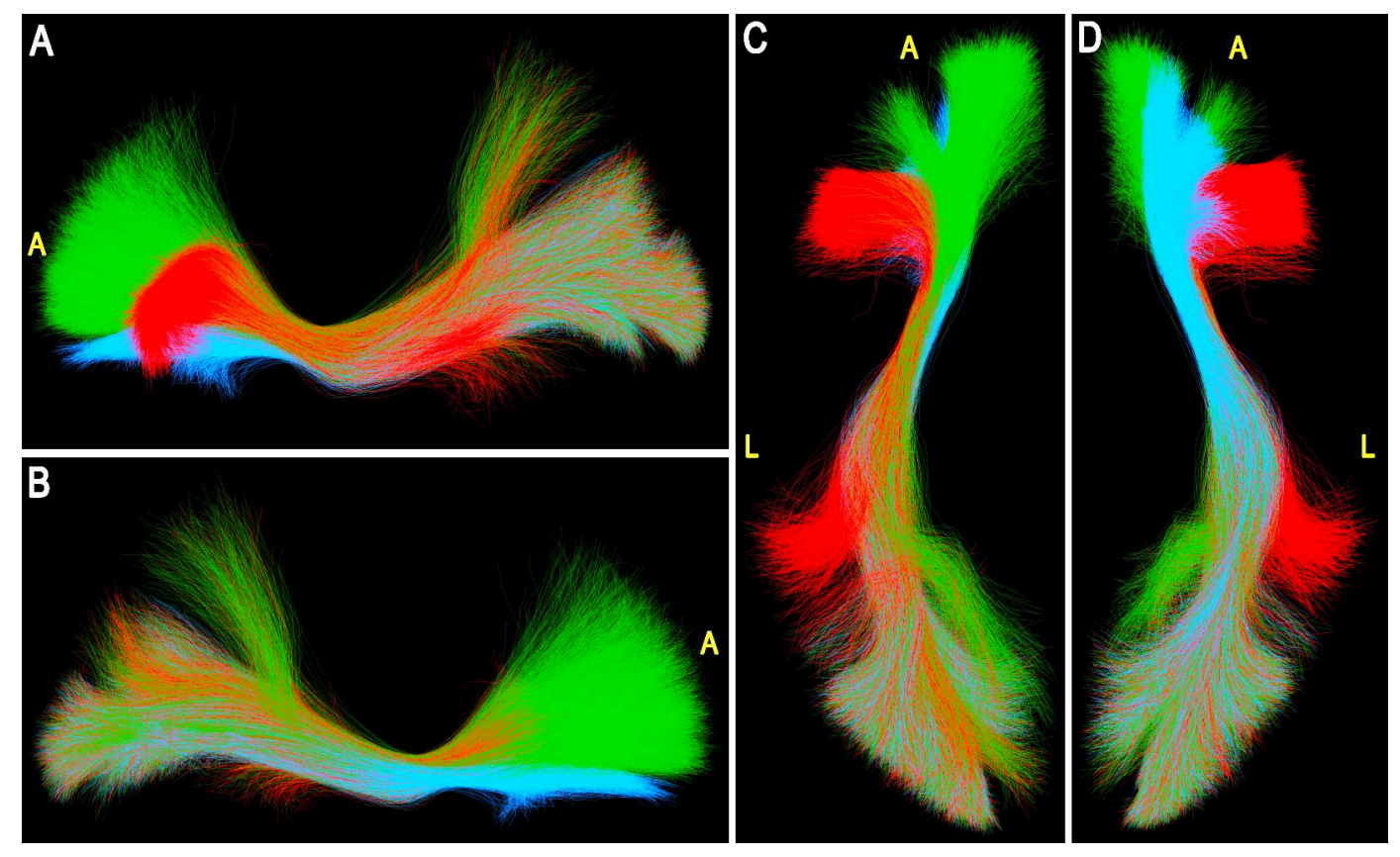




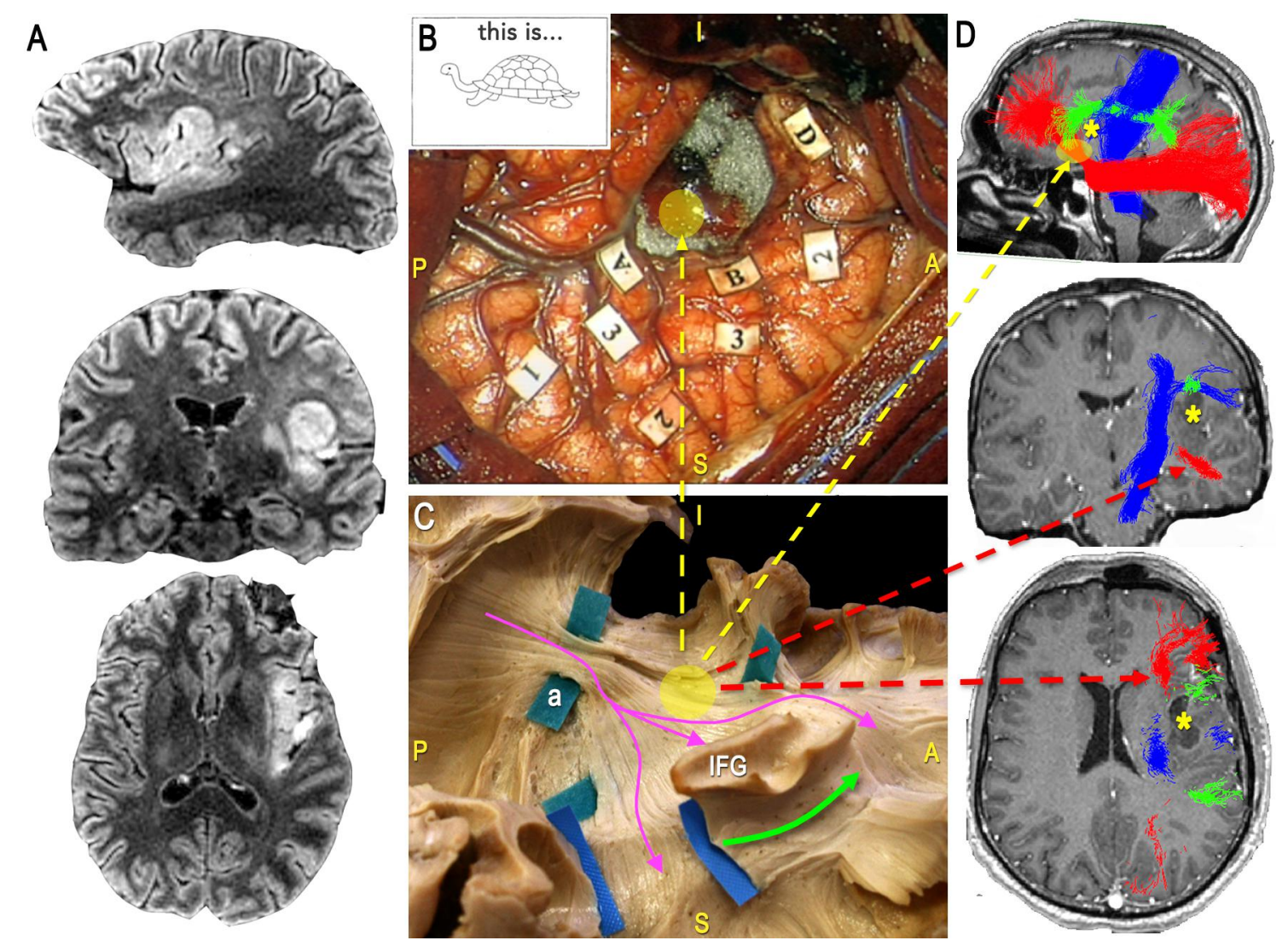

